\title{
Estabilidade e Mudança nas Práticas Educativas Maternas e Paternas ao Longo dos Anos Pré-Escolares da Criança
}

\author{
Stability and Changes in Mothers and Fathers' Child-Rearing Practices \\ Along Child's Pre-School Years
}

\author{
Angela Helena Marin ${ }^{*}$, , Cesar Augusto Piccinini ${ }^{b}$ \& Jonathan R. H. Tudge ${ }^{c}$ \\ ${ }^{a}$ Universidade Luterana do Brasil, Canoas, Brasil \\ ${ }^{b}$ Universidade Federal do Rio Grande do Sul, Porto Alegre, Brasil \\ $\&{ }^{c}$ University of North Carolina at Greensboro, Greensboro, USA
}

\begin{abstract}
Resumo
O presente estudo teve como objetivo examinar a estabilidade e mudança nas práticas educativas maternas e paternas, em particular o uso de práticas indutivas, coercitivas e de não interferência aos 24,36 e 72 meses de vida da criança. Participaram 24 mães e pais que tinham um único filho e residiam na região metropolitana de Porto Alegre. Quando as crianças completaram 24 e 72 meses de vida, os participantes responderam a uma entrevista para a avaliação das práticas educativas. Já aos 36 meses, as famílias foram observadas durante o almoço, quando as práticas educativas também foram investigadas. ANOVAs de medidas repetidas indicaram diferenças quanto às práticas indutivas maternas entre os 24 e 36 meses, bem como entre as práticas de não interferência entre os 24 e 36 meses e também entre os 36 e 72 meses. Quanto aos pais, as diferenças foram encontradas entre as práticas indutivas aos 24 e 72 meses e entre as práticas de não interferência aos 24 e 36 meses e aos 36 e 72 meses. Os resultados sugerem que tanto estabilidade quanto mudanças estão presentes nas práticas educativas parentais ao longo do desenvolvimento da criança. Isto pode estar associado ao fato de que à medida que ela cresce, expectativas e comportamentos parentais acabam sendo adaptados às novas habilidades e demandas infantis.
\end{abstract}

Palavras-chave: Parentalidade; Práticas educativas maternas e paternas.

\begin{abstract}
The present study had as its main objective to examine stability and change in mothers and fathers' childrearing practices, in particular the use of inductive, coercive and non-interference practices with 24, 36 and 72 month-old children. Twenty four mothers and fathers who had an only child and lived in the metropolitan area of Porto Alegre/RS participated in the study. When the children were 24 and 72 months old, the participants were interviewed about their child-rearing practices. When the children were 36 months old, they and their families were observed during lunch time, with focus on child-rearing practices. Repeated measures of ANOVA indicated differences in maternal inductive practices between 24 and 36 months old and differences in non-interference practices both between 24 and 36 as well as 36 and 72 months old. Concerning the fathers, differences were found between inductive practices at 24 and 72 months old and among non-interference practices at 24 and 36 months old as well as at 36 and 72 months old. The results suggest that both stability and change are found in parents' child-rearing practices over the course of children's development. Changes are related to the fact that parents' expectations and behaviors reflect their children's new abilities and demands.

Keywords: Parenthood; Mothers and Fathers' Child-Rearing Practices
\end{abstract}

O tema das práticas educativas parentais tem sido pesquisado sistematicamente desde o início do século XX, mas ainda se constitui como relevante devido às contínuas mudanças que ocorrem nos contextos social e fami-

\footnotetext{
"Endereço para correspondência: Av. Lavras, 654/601, Porto Alegre, RS, Brasil, CEP 90460-040. E-mail: ahmarin@hotmail.com

Artigo derivado da tese de doutorado de Angela Helena Marin, realizada sob a supervisão de Cesar Augusto Piccinini e Jonathan Tudge, apresentada no Programa de Pós-Graduação em Psicologia da Universidade Federal do Rio Grande do Sul, Porto Alegre, RS
}

liar. Uma das questões importantes quanto às práticas educativas parentais diz respeito não só à consistência entre a mãe e o pai, mas também à estabilidade ou mudança destas práticas ao longo do desenvolvimento infantil (Peisner, 1989; Pettit, Keiley, Laird, Bates, \& Dodge, 2007). Mesmo que os pais prezem pela estabilidade de suas práticas, ao longo do desenvolvimento da criança surge a necessidade de novas práticas educativas, pois as antigas expectativas e regras podem não ser mais aplicáveis (Blandon \& Volling, 2008; Grusec \& Lytton, 1988).

Maccoby (1980) sugeriu que quanto maior a idade da criança, mais os pais usariam estratégias como explica- 
ções, argumentações e gratificações, ao mesmo tempo em que demonstrariam menos afeto físico, tornando-se menos protetores e dedicando menos tempo aos seus filhos ${ }^{1}$. A autora destacou que, com o passar dos anos, a punição pelo isolamento e a repreensão física tenderiam a ser desvalorizadas, enquanto a privação de alguns privilégios passaria a ganhar maior importância e o elogiar seria visto como mais efetivo que o punir.

À medida que a criança cresce, o controle e a orientação dos pais começam a colidir com as exigências de autonomia dos filhos, o que leva a modificações no modo como os pais tendem a interagir com eles (Minuchin, 1980). Hudson e Rapee (2001), em um estudo que avaliou 95 díades mãe-criança australianas, apontaram que as mães tendiam a se envolver menos com seus filhos à medida que estes cresciam. Nessa mesma direção, Oliveira, Frizzo e Marin (2000) revelaram que haveria uma diminuição do controle parental, principalmente sobre alguns cuidados de rotina, como, por exemplo, os cuidados físicos, assim como uma diminuição do tempo dedicado aos filhos.

Outro fator a ser considerado como importante na determinação das práticas educativas utilizadas pelos pais é a atribuição de maior ou menor competência ou responsabilidade aos filhos, o que pode estar relacionado com a idade ou a maturidade conferida a eles (Daggett, O'Brien, Zanolli, \& Peyton, 2000). Conforme Dix, Ruble e Zambarano (1989), quando os pais acreditam que o mau comportamento do filho se dá em função da falta de competência da criança, eles tendem a agir de modo mais indutivo do que quando acreditam que ele seja competente e, consequentemente, responsável pelos seus atos. Além disso, a aquisição de novas habilidades por parte da criança também deve ser considerada. À medida que as crianças se tornam mais velhas, os pais tendem a esperar mais delas e a acreditar que possuem mais conhecimento, controle e responsabilidade sobre seus comportamentos negativos. Por exemplo, à medida que a criança se torna assertiva, ela começa a negociar sua independência em relação aos pais, defender seus direitos e expressar seus pensamentos, sentimentos e crenças de forma direta (Falcone, 2001). Neste contexto, cabe aos pais explicar à criança porque ela deve cooperar quando necessário, tentar persuadi-la ou buscar alternativas que ela possa aceitar melhor (Crockenberg \& Littman, 1990).

Além dos fatores mencionados, o desenvolvimento cognitivo da criança também é importante para sua relação com seus pais. À medida que a criança se torna capaz de resolver problemas, os pais tendem a reagir de forma mais indutiva, o que tende a levar a comportamentos prósociais por parte da criança, como pensar sobre as consequências de seu comportamento para os demais (Maccoby,

\footnotetext{
1 Os termos "filho" e "filhos" serão utilizados, no presente estudo, para se referir tanto a crianças do sexo masculino como feminino.
}

1980). Outro fator de influencia na determinação do tipo de prática educativa a ser considerado é o desenvolvimento da linguagem da criança. Mussen Conger, Kagan e Huston (1995) salientaram que a partir do momento em que a criança adquire a habilidade de se comunicar, os pais tendem a fazer maior uso de orientações verbais e explicações racionais. Conseguir compreender e se manifestar verbalmente também foram apontados como um componente que tende a diminuir a interação física entre pais e filhos (Kuczynski, Kochanska, Radke-Yarrow, \& Girnius-Brown, 1987).

Alguns estudos também destacaram que as mudanças nas práticas educativas parentais decorreriam das variações desenvolvimentais, como a passagem da infância para a adolescência. Por exemplo, McNally, Eisenberg e Harris (1991) examinaram 32 mães americanas por meio de instrumentos padronizados quando seus filhos tinham entre sete e 16 anos e indicaram que os pais tendiam a exercer menor controle, usar menos supervisão e enfatizar a autonomia e a independência à medida que seus filhos se tornavam adolescentes. Além disso, eles tendiam a usar mais instruções verbais com fins disciplinares e explicações racionais e maior restrição de privilégios, bem como demonstravam menos afeto físico e ficavam menos tempo com seus filhos. Nessa mesma direção, Paikoff e Brooks-Gunn (1991) e Pettit et al. (2007) demonstraram que o envolvimento parental, a supervisão e o monitoramento tendiam a diminuir quando a criança se aproximava da adolescência e destacaram que diferenças também ocorriam no ambiente familiar, que faziam com que tanto o comportamento dos pais quanto dos filhos se ajustassem mutuamente.

Já a pesquisa desenvolvida por Roberts, Block e Block (1984), com uma amostra americana de 65 mães e 42 pais que responderam a um instrumento padronizado quando seus filhos tinham três e 12 anos de idade, indicou que havia uma considerável estabilidade nas orientações das práticas educativas parentais desde o início da infância até o início da adolescência. Os autores pontuaram que as mudanças ocorridas nestas práticas parecem estar mais associadas a fases do desenvolvimento, como a puberdade, do que à idade per se e salientaram que o comportamento dos pais tendia a refletir as necessidades e competências das crianças, bem como aquelas presentes em suas próprias expectativas.

Percebe-se que, por um lado, as famílias tendem a manter um padrão de relacionamento estável ao longo do tempo, por outro, o desenvolvimento da criança pode agir fortemente para que se produzam mudanças, requerendo dos pais e da própria criança formas diferentes de interação. Maccoby (1984) questiona se essas duas posições seriam compatíveis, assinalando que de certa forma sim, pois algumas práticas tendem a mudar devido ao desenvolvimento da criança, embora o estilo como os pais costumam lidar com seus filhos tenda a se manter estável. O que parece ocorrer, segundo McNally et al. (1991), é uma 
relativa estabilidade quanto aos valores parentais, enquanto algumas práticas específicas, que estão a favor destes, tendem a mudar conforme a idade da criança, devido à aquisição de novas habilidades e maior autonomia. Nesse sentido, o objetivo do presente estudo foi investigar a esta-bilidade e mudança nas práticas educativas maternas e paternas, em particular o uso de práticas indutivas, coercitivas e de não interferência aos 24,36 e 72 meses de vida da criança.

\section{Método}

\section{Participantes}

Participaram deste estudo 24 famílias, constituídas pela mãe, pai e seu primeiro filho, que tinha 24 meses de idade no início do presente estudo. As mães tinham idade média de 29,5 anos $(D P=6,8)$ e os pais 27,2 anos $(D P=5,7)$. Em termos de escolaridade das mães, houve uma variação entre o ensino fundamental incompleto (22\%) e completo (12\%), ensino médio incompleto (12\%) e completo (25\%) e ensino superior incompleto (12\%) e completo (17\%). A escolaridade dos pais também variou entre o ensino fundamental incompleto (34\%) e completo $(8 \%)$, ensino médio incompleto (4\%) e completo (29\%) e ensino superior incompleto (21\%) e completo (4\%). Já quanto ao sexo da criança, $54 \%$ eram meninos e $46 \%$ meninas. Em relação ao nível socioeconômico da família, que foi calculado com base no nível educacional e ocupacional das mães e dos pais (Hollingshead, 1975, adaptado por Tudge \& Frizzo, 2002), a variação ocorreu entre o nível baixo (21\%), o médio-baixo (21\%), o médio $(29 \%)$, o médio-alto $(21 \%)$ e o alto $(8 \%)^{2}$.

A amostra foi selecionada dentre os participantes do Estudo Longitudinal de Porto Alegre: Da Gestação à Escola ([ELPA $\left.{ }^{3}\right]$, Piccinini, Tudge, Lopes, \& Sperb, 1998). Este estudo iniciou acompanhando 81 gestantes que não apresentavam intercorrências clínicas e que estavam esperando seu primeiro filho, juntamente com seus maridos/companheiros. Os bebês, nascidos a termo, também não apresentavam problemas de saúde. Os participantes

${ }^{2}$ Esse cálculo considera quatro fatores: educação, ocupação, estado civil e sexo, das mães e dos pais. Desses fatores derivam as seguintes categorias: Nível $1-$ trabalhadores sem habilidades, empregados domésticos; Nível 2 - operadores de máquinas, trabalhadores com poucas habilidades; Nível 3 - trabalhadores com habilidades, auxiliares de escritório, balconistas; Nível 4 - proprietários de empresas de médio porte, técnicos; Nível 5 - proprietários de grandes empresas e profissionais de nível superior.

${ }^{3} \mathrm{O}$ estudo envolve várias fases de coletas de dados realizadas desde a gestação até os oito anos de vida das crianças (gestação, 3o, $8^{\circ}, 12^{\circ}, 18^{\circ}, 24^{\circ}, 36^{\circ}$ mês e $6^{\circ}$, $7^{\circ}$ e $8^{\circ}$ ano de vida da criança). O objetivo é investigar tanto os aspectos subjetivos e comportamentais das interações iniciais mãe-pai-bebê, assim como o impacto de fatores iniciais do desenvolvimento nas interações familiares, no comportamento social de crianças pré-escolares e na transição para a escola de ensino fundamental. representavam várias configurações familiares (nucleares, uniparentais ou recasados), diferentes idades (adultos e adolescentes) e escolaridade e níveis socioeconômicos variados. Para fins do presente estudo, foram escolhidos todos os casos cujas mães e pais viviam em união estável, tendo ou não oficializado a união, e que tinham dados completos nas coletas realizadas aos 24,36 e 72 meses de vida da criança, totalizando as 24 famílias descritas acima.

\section{Delineamento, Procedimentos e Instrumentos}

Foi utilizado um delineamento longitudinal (Mussen, 1992) para se investigar a estabilidade e mudança nas práticas educativas maternas e paternas, particularmente o uso de práticas indutivas, coercitivas e de não interferência aos 24, 36 e 72 meses de vida da criança. De acordo com Mussen (1992), os delineamentos longitudinais permitem avaliar a estabilidade dos comportamentos ao longo de um determinado período de tempo, além de possibilitar identificar as mudanças que ocorrem e investigar por que alguns comportamentos são variáveis.

No presente estudo, foram consideradas apenas algumas das diversas fases de coleta de dados do ELPA detalhadas em Piccinini et al. (1998). Inicialmente, quando as gestantes estavam no terceiro trimestre de gestação, elas preencheram a Ficha de Contato Inicial (Grupo de Interação Social, Desenvolvimento e Psicopatologia [GIDEP], 1998a), informando alguns dados sociodemográficos sobre o casal. Os casais que preencheram os critérios do estudo (estar esperando o primeiro filho, estar no terceiro trimestre de gestação e não apresentar complicações físicas durante a gravidez) foram contatados, posteriormente, por telefone para agendar uma visita domiciliar. Durante essa visita, foram preenchidos o Termo de Consentimento Livre e Esclarecido e a Entrevista de Dados Demográficos (GIDEP, 1998b). Quando as crianças completaram 24 meses, as famílias foram novamente contatadas por telefone e convidadas a comparecer ao Instituto de Psicologia da Universidade Federal do Rio Grande do Sul. Nessa ocasião, foi realizada a Entrevista sobre Práticas Educativas Parentais (Piccinini \& Alvarenga, 2000), para a avaliação das práticas educativas utilizadas pelas mães e pelos pais ${ }^{4}$. Aos 36 meses de vida das crianças, as famílias foram novamente contatadas para agendar uma visita em suas residências para a realização da Observação das Atividades Cotidianas Familiares (Tudge, Sidden, \& Putnam, 1990). Essa sessão de observação incluiu a filmagem do momento de almoço da família e foi utilizada para a avaliação das práticas educativas observadas na interação das díades mãe-crian-

\footnotetext{
${ }^{4}$ Parte desses dados já foi analisada por Alvarenga, Piccinini, Frizzo, Lopes e Tudge (2009), em um estudo intitulado "Estabilidade e Mudanças nas Práticas Educativas de Mães e Pais dos 18 para os 24 Meses de Vida da Criança".
} 
ça e pai-criança. O contexto de alimentação foi escolhido por se constituir em uma situação durante a qual tendem a ocorrer diversos impasses na interação com a criança (ex.: a criança pode se recusar a comer, não se comportar adequadamente ou querer sair da mesa), sendo, portanto, uma oportunidade rica para se examinar as práticas educativas comumente utilizadas pelas mães e pelos pais (Bastos, Zannon, \& Alcântara, 2003; Crockenberg \& Littman, 1990; Huang, Teti, Caughy, Feldstein, \& Genevro, 2007). Já aos 72 meses de vida da criança, as famílias foram contatadas para uma nova coleta de dados, que consistiu em uma visita em suas residências, situação em que se reaplicou a Entrevista sobre Práticas Educativas Parentais, utilizada para reavaliar as práticas educativas utilizadas pelas mães e pelos pais.

A Entrevista sobre Práticas Educativas Parentais foi originalmente elaborada a partir das propostas de Hart, Ladd e Burleson (1990) e Piccinini, Maggi e Carro (1993) e está estruturada com questões abertas que buscam investigar as práticas educativas utilizadas pela mãe e pelo pai na orientação do comportamento de suas crianças. Ela envolve seis situações cotidianas, as quais têm sido apontadas pela literatura como propícias à ocorrência de práticas educativas e que podem envolver comportamentos infantis inadequados e de desobediência, como, por exemplo, quando a criança recusa-se a comer, a entrar/sair do banho; a trocar de roupa; a dormir à noite; a ficar com outra pessoa, que não seja a sua mãe ou seu pai e quando faz birra ao receber um não dos pais. Aos 72 meses, duas alterações foram feitas no sentido de acompanhar a etapa do desenvolvimento das crianças. A situação envolvendo a recusa em trocar de roupa, foi adaptada para insistir em vestir algumas peças de roupa prediletas; e a situação recusar-se a ficar com outra pessoa, foi modificada para recusar-se a ir/voltar da creche/ escola. Com base em Hoffman $(1970,1975,1994)$ e considerando as sugestões de Alvarenga e Piccinini (2003), as práticas educativas foram classificadas como práticas indutivas, que envolviam as subcategorias negocia/troca, explica/fala, explica baseado em convenções/consequências, organiza o ambiente/forma hábito e comanda sem coerção; e práticas coercitivas, que reuniam as subcategorias punição, ameaça, coação física e punição física. Além dessas categorias, foi necessário incluir uma terceira, denominada não interferência, que envolveu as subcategorias não se intromete, segue o ritmo da criança e cede à vontade da criança. Ainda, algumas estratégias educativas não se enquadraram nas categorias mencionadas e foram denominadas outras, incluindo as subcategorias pede ajuda para outra pessoa e engana (Piccinini, Frizzo, Alvarenga, Lopes, \& Tudge, 2007).

Todas as entrevistas foram gravadas em áudio, transcritas para fins de análise e examinadas por meio da análise de conteúdo quantitativa (Bardin, 1977; Laville \& Dionne, 1999), o que permitiu investigar a frequência de respostas das práticas educativas maternas e paternas.
Dois juízes classificaram separadamente $20 \%$ das entrevistas sobre práticas educativas maternas e paternas nas diferentes situações avaliadas, considerando as três principais categorias de práticas educativas ${ }^{5}$. O índice de fidedignidade entre os juízes foi calculado utilizando-se o Coeficiente Kappa e atingiu 0,82 para as mães e 0,88 para os pais. Esses índices, são considerados excelentes, de acordo com Robson (1995) ${ }^{6}$.

A análise das práticas educativas maternas e paternas durante a sessão de observação do almoço foi realizada conforme os critérios da análise de eventos, sugeridos por Fagundes (1985). Para fins de análise, considerou-se como evento, o conjunto de comportamentos e verbalizações envolvendo cada uma das categorias de práticas educativas, separadamente para a mãe e para o pai. A definição das categorias utilizadas na análise dos eventos foi a mesma utilizada para análise das entrevistas referidas acima, com pequenas adaptações para considerar as práticas educativas observadas em vez dos relatos sobre elas, como ocorreu durante as entrevistas (ex.: indução: "Te dou mais batata frita depois que tu comer o papá"; coerção: "Come, senão tu não vai sair depois"; não interfere: "Não quer mais? Não toma então, deixa o copo aqui"). Para o cálculo da fidedignidade, dois juízes classificaram, separadamente, $20 \%$ das descrições da observação da interação do almoço nas três principais categorias de práticas educativas: indutivas, coercitivas e de não interferência. O índice entre os juízes foi calculado utilizando-se o Coeficiente Kappa, e o valor médio obtido para as mães foi de 0,95 , enquanto para os pais foi de 0,90 , índices considerados excelentes, segundo Robson (1995).

\section{Resultados}

Para examinar a estabilidade e mudança nas práticas educativas maternas e paternas ao longo dos 24,36 e 72 meses de vida da criança, utilizou-se a Análise de Variância (ANOVA) de medidas repetidas, valendo-se das comparações múltiplas de Bonferroni.

\section{Práticas Educativas Maternas}

A Tabela 1 apresenta o valor médio, o desvio padrão e o nível de significância das práticas educativas maternas utilizadas entre os 24, 36 e 72 meses de vida da criança.

AANOVA revelou uma diferença significa para as práticas educativas indutivas ao longo dos três momentos examinados $(p<0,004)$. As comparações múltiplas de Bonferroni revelaram que entre os 24 e os 36 meses de 
Marin, A. H., Piccinini, C. A. \& Tudge, J. R. H. (2011). Estabilidade e Mudança nas Práticas Educativas Maternas e Paternas ao Longo dos Anos Pré-Escolares da Criança.

Tabela 1

Média, Desvio Padrão (entre parênteses) e Nivel de Significância (p) da Variância das Práticas Educativas Maternas entre os 24, 36 e 72 Meses de Vida da Criança (n=24)

\begin{tabular}{lrrrr}
\hline Práticas Maternas & $24 \mathrm{~m}$ & $36 \mathrm{~m}$ & $72 \mathrm{~m}$ & $\mathrm{p}$ \\
\hline Indutivas & $4,75(2,51)$ & $10,29(6,63)$ & $5,96(3,25)$ & 0,004 \\
Coercitivas & $2,96(1,78)$ & $2,46(3,15)$ & $4,17(2,81)$ & 0,04 \\
Não interferência & $2,21(1,38)$ & $0,71(0,95)$ & $2,83(1,71)$ & 0,001 \\
\hline
\end{tabular}

vida da criança há uma diferença significativa $(p<0,05)$, indicando que as mães são mais indutivas aos 36 meses do que aos 24 meses. Também se destaca uma diferença marginalmente significativa entre os 36 e 72 meses $(p<0,058)$, indicando uma tendência de as mães usarem mais práticas indutivas educativas quando seus filhos apresentam menor idade

Já quanto às práticas coercitivas, a ANOVA também revelou uma diferença significativa no período examinado $(p<0,04)$, mas as comparações múltiplas de Bonferroni falharam ao apontar em que momentos elas ocorreram. $\mathrm{O}$ menor valor de $p$ encontrado entre os momentos analisados foi de 0,10 entre os 36 e os 72 meses, indicando apenas uma tendência de as mães usarem mais práticas coer- citivas com seus filhos de maior idade. Uma explicação para a ausência de diferença significativa no post hoc é que o teste escolhido é bastante conservador (Olejnik, Li, Supattathum, \& Huberty, 1997) e, por vezes, não consegue confirmar os resultados significativos da ANOVA, ao não identificar o momento em que ocorreram as diferenças.

Por fim, a ANOVA revelou diferença significativa $(p<0,001)$ para as práticas de não interferência. As comparações múltiplas de Bonferroni revelaram diferença significativa entre os 24 e 36 meses $(p<0,001)$ e os 36 e 72 meses de vida da criança $(p<0,001)$, indicando que tais práticas foram menos utilizadas quando a criança tinha 36 meses do que quando ela tinha 24 e 72 meses.

Tabela 2

Média, Desvio Padrão (entre parênteses) e Nivel de Significância (p) da Variância das Práticas Educativas Paternas aos 24, 36 e 72 Meses de Vida da Criança ( $n=24)$

\begin{tabular}{lrrrr}
\hline Práticas Paternas & $24 \mathrm{~m}$ & $36 \mathrm{~m}$ & $72 \mathrm{~m}$ & $\mathrm{p}$ \\
\hline Indutivas & $3,00(1,47)$ & $5,79(5,54)$ & $5,50(3,61)$ & 0,04 \\
Coercitivas & $2,17(1,58)$ & $2,38(2,70)$ & $3,50(2,52)$ & 0,04 \\
Não interferência & $2,75(1,65)$ & $0,21(0,51)$ & $2,83(2,14)$ & 0,001 \\
\hline
\end{tabular}

\section{Práticas Educativas Paternas}

A Tabela 2 apresenta o valor médio, o desvio padrão e o nível de significância das práticas educativas paternas utilizadas entre os 24,36 e 72 meses de vida da criança.

ANOVA revelou diferenças significativas em relação às práticas educativas indutivas paternas utilizadas nos três momentos examinados $(p<0,04)$. As comparações múltiplas de Bonferroni, revelaram uma diferença significativa $(p<0,003)$ entre as práticas indutivas utilizadas pelos pais aos 24 meses e aos 72 meses de vida da criança, indicando que eles foram mais indutivos quando seus filhos apresentavam maior idade.

Quanto às práticas coercitivas, foram verificadas diferenças significativas ao longo do período examinado $(p<0,04)$, mas as comparações múltiplas de Bonferroni falharam em apontar onde elas se encontravam. O menor valor de $p$ encontrado entre os momentos analisados foi de 0,09 entre os 24 e os 72 meses, indicando que os pais tenderam a ser mais coercitivos quando seus filhos tinham maior idade.
Por fim, a ANOVA revelou uma diferença significativa para as práticas de não interferência $(p<0,001)$. As comparações múltiplas de Bonferroni revelaram diferença significativa aos 24 e $36(p<0,001)$, bem como aos 36 e 72 meses de vida da criança $(p<0,001)$ indicando que os pais utilizaram menos práticas de não interferência quando a criança tinha 36 meses do que quando tinha 24 e 72 meses de vida.

\section{Diferenças nas Práticas Educativas Maternas e Paternas}

Para examinar as diferenças entre as mães e os pais quanto às práticas educativas, utilizou-se o teste nãoparamétrico Wilcoxon. Para tanto, foi calculado o valor do delta entre os momentos examinados $(36 \mathrm{~m}-24 \mathrm{~m}$; $72 \mathrm{~m}-24 \mathrm{~m} ; 72 \mathrm{~m}-36 \mathrm{~m}$ ) para cada uma das categorias de práticas educativas consideradas. A Tabela 3 apresenta a média, o desvio padrão e o nível de significância dos deltas das práticas educativas maternas e paternas. 
Tabela 3

Média e Desvio Padrão (entre parênteses) dos Deltas das Práticas Educativas Maternas e Paternas entre os 24, 36 e 72 Meses de Vida da Criança $(n=24)$

\begin{tabular}{lrrr}
\hline & Maternas & Paternas & $p$ \\
\hline Práticas entre & $36-24 m$ & & \\
Indutivas & $5,54(7,68)$ & $2,79(6,08)$ & 0,14 \\
Coercitivas & $-0,50(3,16)$ & $0,21(2,22)$ & 0,36 \\
Não interferência & $-1,50(1,67)$ & $-2,04(1,88)$ & 0,23 \\
Práticas entre $72-24$ & & & \\
Indutivas & $1,21(3,20)$ & $2,50(3,24)$ & 0,13 \\
Coercitivas & $1,21(2,79)$ & $1,33(2,85)$ & 0,74 \\
Não interferência & $0,62(1,91)$ & $0,008(1,99)$ & 0,39 \\
Práticas entre $72-36$ & & & \\
Indutivas & $-4,79(8,81)$ & $-0,29(6,37)$ & 0,01 \\
Coercitivas & $1,71(3,72)$ & $1,12(3,03)$ & 0,47 \\
Não interferência & $2,12(1,80)$ & $2,62(2,18)$ & 0,28 \\
& & &
\end{tabular}

Os resultados revelaram apenas uma diferença significativa entre mães e pais, no que diz respeito às práticas indutivas entre os 36 e 72 meses $(p<0,01)$. Isto indica que houve maior redução no uso das práticas indutivas pelas mães $(-4,79)$ do que pelos pais $(-0,29)$ entre esses dois momentos.

\section{Discussão}

Estabilidade e mudanças nas práticas educativas parentais são esperadas quando se considera o processo de socialização infantil, porque, embora exista uma tendência à estabilidade destas práticas, à medida que a criança vai crescendo surge a necessidade de adaptá-las a novas situações e às suas aquisições desenvolvimentais (Grusec \& Lytton, 1988; Hudson \& Rapee, 2001; Maccoby, 1980; Minuchin, 1980; Oliveira et al., 2000; Peisner, 1989; Pettit et al., 2007). Como esperado, os resultados do presente estudo revelaram mudanças nas práticas educativas maternas e paternas ao longo do período considerado. Mais especificamente, as práticas indutivas e de não interferência apresentaram diferenças significativas entre os momentos examinados, enquanto as práticas coercitivas tenderam a se manter estáveis.

Em relação às mudanças encontradas entre as práticas indutivas, destaca-se que tanto as mães quanto os pais tenderam a ser mais indutivos com seus filhos aos $36 \mathrm{e}$ 72 meses. Particularmente, as mães foram mais indutivas aos 36 meses, em comparação aos 24 meses, enquanto os pais foram mais indutivos aos 72 meses, em relação aos 24 meses. Tais resultados sugerem que o uso de práticas indutivas pode estar relacionado à atribuição de maior competência ou responsabilidade aos filhos, o que, por sua vez, pode estar associado com a idade ou a maturidade conferida a eles. À medida que cresce, a criança se torna mais assertiva e começa a negociar sua independência em relação aos pais, defender seus direitos e expressar seus pensamentos, sentimentos e crenças de forma direta (Falcone, 2001), cabendo aos pais lhe explicar porque ela deve cooperar quando necessário, tentar persuadi-la ou buscar alternativas que ela possa aceitar melhor (Alvarenga, Piccinini, Frizzo, Lopes, \& Tudge, 2009; Crockenberg \& Littman, 1990).

Além disso, é importante considerar o desenvolvimento cognitivo da criança, pois, à medida que ela se torna capaz de compreender e resolver problemas, os pais tendem a reagir de forma mais indutiva (Maccoby, 1980). O desenvolvimento da linguagem também é fundamental, porque a partir do momento em que a criança adquire a habilidade de se comunicar, os pais tendem a fazer maior uso de orientações verbais e explicações racionais (Kuczynski et al., 1987; Mussen et al., 1995).

No que diz respeito às práticas de não interferência, tanto as mães como os pais apresentaram diferenças significativas em relação ao seu uso entre os 24 e 36 meses, bem como entre os 36 e 72 meses de vida da criança. Parece que, da mesma forma como ocorre com as práticas indutivas, as práticas de não interferência passam a ser mais frequentes à medida que a criança cresce e talvez estejam associadas ao surgimento da assertividade (Crockenberg \& Littman, 1990). Chama à atenção o fato de a etapa de coleta de dados aos 36 meses de vida da criança ter sido uma sessão de observação. Nesse contexto, as mães e os pais podem ter se sentido imbuídos da necessidade de interagir com a criança, manifestando algum tipo de prática, seja ela indutiva, coercitiva ou outra. Já quando questionados sobre as práticas que costumavam usar, o que foi feito aos 24 e 72 meses, eles podem ter se sentido mais à vontade para simplesmente dizer que não interferiram no comportamento da criança, permitindo que ela mesma o determinasse.

Em contraposição as práticas indutivas e de não interferência, as práticas coercitivas tenderam a manter certa estabilidade ao longo do desenvolvimento da criança. Peisner (1989) já enfatizava que, no processo de socialização infantil, algumas práticas tendem a se manter estáveis enquanto outras não. Talvez esse seja o caso das práticas coercitivas, que pareceram ser mais utilizadas em situações de maior conflito com a criança ou como última alternativa para atingir o objetivo disciplinar almejado, especialmente para algumas circunstâncias, após o uso de práticas indutivas e mesmo de não interferência.

Nesse sentido, frente a situações conflitivas, independentemente da idade da criança, as mães e os pais acabam usando práticas coercitivas, como último recurso. Por exemplo, aos 24 meses de vida da criança, as práticas coercitivas foram mais mencionadas tanto pelas mães como pelos pais na situação de birra, possivelmente devido à característica aversiva desse comportamento. Assim, a prática coercitiva pode estar sendo utilizada com o objetivo de socialização de curto prazo, ou seja, interromper imediatamente o comportamento da criança, que 
precisa obedecer à mãe ou ao pai (Newcombe, 1996/1999; Weber, Viezzer, \& Brandenburg, 2004). Já aos 72 meses, as práticas coercitivas foram mais utilizadas nas situações do banho, o que pode estar refletindo o nível de desenvolvimento da criança, que, nessa idade, prefere determinar os seus próprios comportamentos, inclusive quanto aos horários de suas atividades diárias e quanto ao que prefere fazer. Nesse sentido, Biasoli-Alves e Caldana (1992) referem que a presença dos pais na situação de banho tende a ser mais importante, por tratar-se de um hábito de higiene que precisa ser seguido, embora assinalassem que as decisões geralmente tendem a ser feitas em conjunto com a criança.

Os resultados do presente estudo ainda mostraram muita semelhança entre as práticas educativas maternas e paternas, o que corrobora a literatura (Bentley \& Fox, 1991; Weber, Prado, Viezzer, \& Brandenburg, 2004). Com exceção das práticas indutivas, que, no caso das mães, tiveram uma redução significativa entre os 36 e 72 meses de vida da criança, as demais comparações entre mães e pais, não se mostraram significativas. Contudo, mesmo em relação às praticas indutivas, a sua incidência entre as mães e os pais foi praticamente a mesma aos 72 meses, mas, no caso das mães, houve uma redução muito acentuada em relação ao 36 meses, quando as práticas indutivas tiveram seu ápice, enquanto no caso dos pais os valores foram muito semelhantes nos dois momentos.

Juntos esses achados mostram que tanto estabilidade quanto mudanças nas práticas educativas parentais tendem a estar presentes ao longo do desenvolvimento da criança, porque, à medida que a criança cresce, antigas expectativas e regras parentais mudam para se adaptar as novas demandas e habilidades infantis. Ao mesmo tempo, é plausível pensar que apesar das eventuais mudanças nas práticas educativas, muitas delas parecem preservar os valores parentais. Assim, é possível que as famílias procurem manter um padrão estável de relacionamento ao longo do tempo, mas, por outro lado, o desenvolvimento infantil tende a incitar mudanças, requerendo dos pais e da própria criança formas diferentes de interação.

Cabe ressaltar algumas limitações do presente estudo, como o pequeno número de participantes, que pode ter reduzido o poder estatístico das análises e os intervalos diferentes de tempo entre os momentos investigados. Além disto, o uso de dois instrumentos diferentes pode ter contribuído para que se captasse aspectos específicos das práticas educativas, exacerbando eventuais diferenças. Embora inconsistências entre os dados obtidos por meio de entrevista e observação já tenham sido destacadas pela literatura, alguns autores (Dessen \& Lewis, 1998; Karreman, van Tuijl, van Aken, \& Dekovic, 2008) sugerem que ambos os procedimentos podem ser complementares. Estudos observacionais têm sido apontados como vitais para investigar se o comportamento relatado corresponde ao comportamento observado, embora grande parte das pesquisas sobre práticas educativas ainda utilizem, com maior frequência, os relatos parentais. Em um estudo recente que investigou se as práticas que as mães relatavam estavam de acordo com o que elas de fato empregavam, Wilson, Gardner, Burton e Leung (2007) afirmaram que os relatos e a observação, mesmo que proporcionem perspectivas diferentes sobre o comportamento parental, podem juntos oferecer uma melhor compreensão sobre as práticas educativas parentais. $\mathrm{Na}$ verdade, a utilização de múltiplos métodos contribui para estabelecer uma validade convergente, comparando os resultados derivados de duas ou mais medidas de avaliação do mesmo construto (Harkness et al., 2006). Conceitualmente, a integração de diferentes perspectivas subjacentes a cada método pode ajudar a interpretar resultados complexos e a entendê-los a partir de um referencial mais amplo.

Apesar dessas limitações, o presente estudo contribui para área da psicologia do desenvolvimento ao demonstrar que algumas das práticas educativas maternas e paternas, particularmente as indutivas e de não interferência, tendem a variar mais ao longo do crescimento da criança, enquanto outras, como as coercitivas, tendem a apresentar menos variações. Os resultados também destacam a complexidade de investigar as práticas educativas, tendo em vista os inúmeros fatores, culturais, sociais, familiares e individuais que contribuem para a sua expressão. Neste contexto, muitas vezes é difícil responder as demandas atuais de mães e pais que buscam orientação para lidar com os filhos e que vivem frequentemente um processo de insegurança frente ao processo de socialização dos mesmos. Assim sendo, além de escutar e observar as próprias mães e pais, muitos outros estudos, preferencialmente longitudinais, precisam ser feitos para que se possa compreender melhor a estabilidade e mudanças nas práticas educativas ao longo do desenvolvimento da criança.

\section{Referencias}

Alvarenga, P., \& Piccinini, C. (2003). Práticas educativas maternas e a interação entre mães e crianças com problemas de externalização. Aletheia, 17/18, 7-20.

Alvarenga, P., Piccinini, C. A., Frizzo, G. B., Lopes, R. C. S., \& Tudge, J. R. H. (2009). Estabilidade e mudanças nas práticas educativas de mães e pais dos 18 para os 24 meses de vida da criança. Interação em Psicologia, 13(2), 253-262.

Bardin, L. (1977). Análise de conteúdo (L. Reto \& A. Pinheiro, Trads.). São Paulo, SP: Edições 70.

Bastos, A. C. S., Zannon, C. M. L. C., \& Alcântara, M. A. R. (2003). Cultura e desenvolvimento: Uma estratégia de análise a partir de um estudo longitudinal de famílias em Salvador, Bahia. Revista Brasileira de Crescimento e Desenvolvimento Humano, 13(2), 12-30.

Bentley, K. S., \& Fox, R. A. (1991). Mothers and fathers of young children: Comparison of parenting styles. Psychological Reports, 69, 320-322.

Biasoli-Alves, Z. M., \& Caldana, R. H. L. (1992). Práticas educativas: A participação da criança na determinação do seu dia-a-dia. Psicologia: Teoria e Pesquisa, 8(2), 231-242. 
Blandon, A. Y., \& Volling, B. L. (2008). Parental gentle guidance and children's compliance within the family: A replication study. Journal of Family Psychology, 22(3), 355-366.

Crockenberg, S., \& Littman, C. (1990). Autonomy as competence in 2-year olds: Maternal correlates of child defiance, compliance and self-assertion. Developmental Psychology, 26(6), 961-971.

Daggett, J., O’Brien, M., Zanolli, K., \& Peyton, V. (2000). Parents' attitudes about children: Associations with parental life histories and child-rearing quality. Journal of Family Psychology, 14(2), 187-199.

Dessen, M. A., \& Lewis, C. (1998). Como estudar a família e o pai? Paidéia, 8(14/15), 105-121.

Dix, T., Ruble, D. N., \& Zambarano, R. J. (1989). Mothers' implicit theories of discipline: Child effects, parent effects, and the attribution process. Child Development, 60, 13731391.

Fagundes, A. J. (1985). Descrição, definição e registro do comportamento. São Paulo, SP: Edicom.

Falcone, E. O. (2001). Uma proposta de um sistema de classificação das habilidades sociais. In H. J. Guilhardi, M. B. B. P. Madi, P. P. Queiroz, \& M. C. Scoz (Eds.), Sobre comportamento e cognição: Expondo a variabilidade (pp. 195-209). Santo André, SP: ESETec.

Grupo de Interação Social, Desenvolvimento e Psicopatologia. (1998a). Ficha de Contato Inicial. Manuscrito não-publicado. Instituto de Psicologia, Universidade Federal do Rio Grande do Sul, Porto Alegre, RS.

Grupo de Interação Social, Desenvolvimento e Psicopatologia. (1998b). Entrevista de Dados Demográficos. Manuscrito nãopublicado. Instituto de Psicologia, Universidade Federal do Rio Grande do Sul, Porto Alegre, RS.

Grusec, J. E., \& Lytton, H. (1988). Socialization and the family. In J. E. Grusec \& H. Lytton, Social development: History, theory and research (pp. 161-210). New York: SpringerVerlag.

Harkness, S., Moscardino, U., Bermudez, M. R., Zylicz, P. O., Welles-Nystrom, B., Blom, M., et al. (2006). Mixed methods in international collaborative research: The experiences of the International Study of Parents, Children, and Schools. Cross Cultural Research: The Journal of Comparative Social Science, 40(1), 65-82.

Hart, C. H., Ladd, G. W., \& Burleson, B. R. (1990). Children's expectations of the outcomes of social strategies: Relations with sociometric status and maternal disciplinary styles. Child Development, 61, 127-137.

Hoffman, M. L. (1970). Conscience, personality, and socialization techniques. Journal of Human Development, 13(2), 90-126.

Hoffman, M. L. (1975). Moral internalization, parental power, and the nature of parent-child interaction. Developmental Psychology, 11, 228-239.

Hoffman, M. L. (1994). Discipline and internalization. Developmental Psychology, 30, 26-28.

Hollingshead, A. B. (1975). Four factor index of social status. Unpublished manuscript, Department of Sociology, Yale University, UK.

Huang, K. Y., Teti, D. M., Caughy, M. B., Feldstein, S., \& Genevro, J. (2007). Mother-child conflict interaction in the toddler years: Behavior patterns and correlates. Journal of Child \& Family Studies, 16, 219-241.
Hudson, J. L., \& Rapee, R. M. (2001). Parent-child interactions and anxiety disorders: An observational study. Behaviour Research and Therapy, 39(12), 1411-1427.

Karreman, A., van Tuijl, C., van Aken, M. A. G., \& Dekovic, M. (2008). Parenting, coparenting, and effortful control in preschoolers. Journal of Family Psychology, 22(1), 30-40.

Kuczynski, L., Kochanska, G., Radke-Yarrow, M., \& GirniusBrown, O. (1987). A developmental interpretation of young children's noncompliance. Developmental Psychology, 23, 799-806.

Laville, C., \& Dionne, J. (1999). A construção do saber: Manual de metodologia da pesquisa em ciências humanas (L. M. Siman, Trad.). Porto Alegre, RS: Artes Médicas.

Maccoby, E. E. (1980). Social development: Psychological growth and the parent-child relationship. New York: Harcourt Brace Jovanovich.

Maccoby, E. E. (1984). Socialization and developmental change. Child Development, 55, 317-328.

McNally, S., Eisenberg, N., \& Harris, J. D. (1991). Consistency and change in maternal child-rearing practices and values: A longitudinal study. Child Development, 62, 190-198.

Minuchin, S. (1980). Um modelo familiar (J. A. Cunha, Trad.). In S. Minuchin, Famílias: Funcionamento e tratamento (pp. 52-69). Porto Alegre, RS: Artes Médicas.

Mussen, P. (1992). Longitudinal study of the life span. In N. Eisenberg (Ed.), Contemporary topics in Developmental Psychology. New York: John Wiley \& Sons.

Mussen, P. H., Conger, J. J., Kagan, J., \& Huston, A. C. (1995). Socialização na família (M. L. G. L. Rosa, Trad.). In P. H. Mussen, J. J. Conger, J. Kagan, \& A. C. Huston, Desenvolvimento e personalidade da criança (3. ed., pp. 429-466). São Paulo, SP: Harbra.

Newcombe, N. (1999). Socialização no cenário da família (C. Bucheveitz, Trad.). In N. Newcombe, Desenvolvimento infantil: Abordagem de Mussen (8. ed., pp. 336-363). Porto Alegre, RS: Artes Médicas. (Original work published 1996)

Olejnik, S., Li, J., Supattathum, S., \& Huberty, C. J. (1997). Multiple testing and statistical power with modified Bonferroni procedures. Journal of Educational and Behavioral Statistics, 22, 389-406.

Oliveira, E. A. de, Frizzo, G. B., \& Marin, A. H. (2000). Atitudes maternas diferenciais para com meninos e meninas de quatro e cinco anos. Psicologia: Reflexão e Crítica, 13(3), 363-371.

Paikoff, R. L., \& Brooks-Gunn, J. (1991). Do parent-child relationships change during puberty? Psychological Bulletin, 110, 47-66.

Peisner, E. S. (1989). To spare or not spare the rod: A culturalhistorical view of child discipline. In J. Valsiner (Ed.), Child development in cultural context (pp. 111-141). Toronto, Canada: Hogrefe and Huber.

Pettit, G. S., Keiley, M. K., Laird, R. D., Bates, J. E., \& Dodge, K. A. (2007). Predicting the developmental course of mother-reported monitoring across childhood and adolescence from early proactive parenting, child temperament, and parents' worries. Journal of Family Psychology, 21(2), 206-217.

Piccinini, C. A., \& Alvarenga, P. (2000). Entrevista sobre Práticas Educativas Parentais. Manuscrito não-publicado, Instituto de Psicologia, Universidade Federal do Rio Grande do Sul, Porto Alegre, RS. 
Piccinini, C. A., Frizzo, G. B., Alvarenga, P., Lopes, R. C. S., \& Tudge, J. R. H. (2007). Práticas educativas maternas e paternas em crianças aos 18 meses de idade. Psicologia: Teoria e Pesquisa, 23(4), 369-378.

Piccinini, C. A., Maggi, A., \& Carro, J. (1993). Strategies used by mothers of German and Italian descent for regulating their children behavior. Paper presented at the XXII biennial meeting of the International Society for the Study of Behavior Development, Recife, PE.

Piccinini, C. A., Tudge, J. R. H., Lopes, R. C., \& Sperb, T. M. (1998). Projeto longitudinal de Porto Alegre: Da gravidez à escola. Manuscrito não-publicado, Curso de Pós-Graduação em Psicologia do Desenvolvimento, Universidade Federal do Rio Grande do Sul, Porto Alegre, RS.

Roberts, G. C., Block, J. H., \& Block, J. (1984). Continuity and change in parents' child-rearing practices. Child Development, 55, 586-597.

Robson, C. (1995). Real word research: A resource for scientist and practiotioner-researchs. Oxford, UK: Blackwell.

Tudge, J. R. H., \& Frizzo, G. F. (2002). Classificação baseada em Hollingshead do nível sócio-econômico das famílias do estudo longitudinal de Porto Alegre: Da gestação à escola. Manuscrito não-publicado, Porto Alegre, RS.

Tudge, J. R. H., Sidden, J., \& Putnam, S. A. (1990). The cultural ecology of young children: Coding manual. Unpublished manuscript, Greensboro, NC.

Weber, L. N. D., Prado, P. M., Viezzer, A. P., \& Brandenburg, A. J. (2004). Identificação de estilos parentais: O ponto de vista dos pais e dos filhos. Psicologia: Reflexão e Crítica, 17(3), 323-331.

Weber, L. N. D., Viezzer, A. P., \& Brandenburg, O. J. (2004). O uso de palmadas e surras como prática educativa. Estudos de Psicologia (Natal), 9(2), 227-237.

Wilson, C., Gardner, F., Burton, J., \& Leung, S. (2007). Maternal attributions and observed maternal behaviour: Are they linked? Behavioural and Cognitive Psychotherapy, 35(2), 165-178. 